УДК 338.2

$10.17213 / 2075-2067-2020-6-185-192$

\title{
НАПРАВЛЕНИЯ РАЗВИТИЯ ВСПОМОГАТЕЛЬНОГО АППАРАТА МЕХАНИЗМА ОЦЕНОЧНО-ПРОГНОЗНОГО МОДЕЛИРОВАНИЯ В ИНТЕГРАЦИОННЫХ СИСТЕМАХ УПРАВЛЕНИЯ РИСКАМИ
}

\author{
(C) 2020 г. Д. В. Корнеев
}

\section{Первый термометровый завод, г. Валдай, Россия}

Цель исследования. Представлен комплекс взаимодополняющих рекомендащий по повышению эффективности современных методов оченочно-прогнозного моделирования риск-систем. Цель - повысить точность оценки и прогноза результатов взаимодействия макроэкономической и микроэкономической среды, что даст возможность долгосрочного конкурентоспособного развития национальной экономики и сочииальной сферы в условиях растущей нестабильности и неопределенности мировой политэкономической cредbl.

Методологическую базу исследования представляют спектральный метод оценки статистических рисков, нечетко-множественное моделирование риск-систем, общий подход моделирования риск-систем на основе нейронных сетей, стратегическое прогнозирование методом аналитических сетей и др.

Результаты исследования. Исследовательская работа содержит комплекс взаимодополняющих рекомендаций по повышению эффективности современных методов оченочнопрогнозного моделирования риск-систем, предложений по расширению вспомогательного инструментария в сторону получения актуальных для интеграциионных систем риск-менеджмента оченочных прогнозов комплексных синергетических риск-прочессов.

Перспективу исследования составляет работа над созданием универсальной методики оценочно-прогнозного моделирования в интеграциинных системах управления рисками.

Ключевые слова: интеграчионные системы управления рисками; моделирование синергетических эффектов; вспомогательный аппарат механизма оченочно-прогнозного моделирования.

\section{DIRECTIONS OF DEVELOPMENT OF THE AUXILIARY APPARATUS OF THE MECHANISM OF ESTIMATION AND FORECAST MODELING IN INTEGRATED RISK MANAGEMENT SYSTEMS}

\section{(C) 2020 D. V. Korneev}

\section{The First Thermometer Plant, Valdai, Russia}

Purpose of research. A set of complementary recommendations for improving the effectiveness of modern methods of estimation and predictive modeling of risk systems is presented. The goal is to improve the accuracy of assessing and forecasting the results of interaction between the macroeconomic and microeconomic environment, which will enable long - term competitive development of the national economy and the social sphere in the context of growing instability and uncertainty in the global political economic environment. 
The methodological basis of the research is represented by the spectral method of statistical risk assessment, fuzzy-multiple modeling of risk systems, a General approach to modeling risk systems based on neural networks, strategic forecasting using analytical networks, etc.

Research result. The research work contains a set of complementary recommendations for improving the effectiveness of modern methods of estimation and predictive modeling of risk systems, as well as proposals for expansion.

The research perspective is the work on creating a universal methodology for estimation and predictive modeling in integrated risk management systems.

Key words: integrated risk management systems; modeling of synergetic effects; auxiliary apparatus of the mechanism of estimation and forecast modeling.

Введение. Необходимость осуществления эффективного оценочно-прогнозного моделирования комплексных риск-систем с возможными синергетическими воздействиями требует не только разработки соответствующих алгоритмов механизма реализации данных направлений в рамках функционирования расширенной интеграционной системы управления рисками, но и развития соответствующего вспомогательного аппарата, позволяющего транспонировать результаты прогрессивных исследований в области математики, эконофизики, информатики в сферу современного риск-менеджмента с целью их прикладного использования при решении комплексных задач по риск-менеджменту.

Основные направления развития вспомогательного аппарата интегрированного механизма управления рисками. В разрезе намеченной проблематики данного исследования можно выделить три взаимосвязанных направления необходимого развития вспомогательного аппарата интегрированного механизма управления рисками:

- развитие вспомогательного аппарата микроуровня (методики идентификационнооценочного процесса рисковых взаимосвязей и взаимодействий);

- развитие вспомогательного аппарата макроуровня (методологии оценки и прогноза комплексных риск-систем и синергетических риск-процессов с учетом особенностей расширенной интеграционной формы предпринимательских структур и соответствующей многоуровневой системы риск-менеджмента);

- развитие вспомогательного аппарата глобального уровня (эффективного модели- рования и управления современной рисковой средой в рамках реализации новой парадигмы управления будущим с использованием интеграционных динамическо-адаптивных систем управления рисками на разных уровнях комплексного воздействия).

Последние два направления являются наиболее проблемными и требуют поиска новых нестандартных решений.

Все многообразие моделей в научных исследованиях в этой области, в принципе, сводится к нескольким методологическим направлениям (с использованием соответствующего вспомогательного математического аппарата), а именно: детерминированные методы, вероятностно-статистические методы (статистические, теоретико-вероятностные, вероятностно-эвристические), нестатистические и комбинированные. Каждая из этих групп несет определенную функциональную направленность в оценочно-прогнозном механизме риск-менеджмента, обладая определенными достоинствами и недостатками (ограничениями) при решении тех или иных задач, поэтому при реализации комплексного механизма многоуровневой системы управления рисками невозможно применение лишь одной или нескольких универсальных методик моделирования, как и, соответственно, невозможно применение лишь «однородного» вспомогательного математического аппарата.

Анализ современных методов прогнозно-оценочного моделирования. К основному вспомогательному аппарату, обеспечивающему представленные выше методологические направления современного оценочно- 
прогнозного моделирования риск-менеджмента, можно отнести:

— линейные и нелинейные функции (использование регрессионного анализа при оценке рисковых связей);

- дифференциальные функции (осуществление прогнозного моделирования динамических систем);

- нечеткие множества (методы анализа риска в условиях неопределенностей нестатистической природы, предназначены для описания неопределенностей параметров риска);

- математический аппарат нейронных и аналитических сетей (когнитивное прогнозное моделирование риск-систем и принятия решений);

- фрактальную теорию (мультифрактальное моделирование рыночных финансовых колебаний, прогрессивная оценка финансовых рисков);

- теории самоорганизации систем, динамического хаоса и бифуркаций (концептуальное моделирование сложных хаотических процессов в рисковой среде);

- распределительные системы, волновую теорию и теорию колебаний (пока не получившие широкого распространения в рискменеджменте методики, однако, по мнению автора, имеющие перспективное значение для развития моделирования в современных условиях нелинейного многовекторного характера динамической рисковой среды) и другие [3].

Для наибольшего эффекта представляется возможным использование комплексных и комбинированных методов, сформированных согласно логико-функциональному принципу с индивидуальным подходом к специфике интегрированных структур, с одной стороны, и системным подходом к решению сложных комплексных (многоуровневых) задач, с другой стороны. К таким сложным задачам относится в первую очередь моделирование риск-систем и присущих им синергетических процессов.

Таким образом, математический аппарат должен быть дополнен инструментарием синергетического моделирования.

Для адаптации основ синергетического моделирования непосредственно в механизм риск-менеджмента в современных исследованиях преимущественно прибегают к инс- трументарию «фрактального броуновского движения» (при моделировании финансовых рисков), а также аппарату нейронных сетей и «нечетких множеств». Применение именно этого математического аппарата при оценке сложных хаотических риск-систем обусловливается возможностью использования множественных условных (неточно определенных) параметров (целей, значений, ограничений) и допущений для построения соответствующих моделей. Например, использование аппарата нейронных сетей может способствовать в том числе определению «нечетких связей» взаимодействия между риск-факторами различных уровней комплексной системы. При этом изначальное конструирование нейронной сети может строиться лишь на слабых допущениях о возможности подобных связей, а формирование входящих и промежуточных слоев переменных для лучшей систематизации должно соответствовать принципу деления факторов (нейронов) по интеграционным уровням воздействия и целевым спецификациям. В качестве выходных данных сети могут использоваться сбалансированные показатели функционирования расширенного интеграционного образования (также систематизированные по многоуровневому принципу) либо информация о последствиях реализации (или коррекции) соответствующих рисков (при наличии необходимого объема подобных статистических данных.

Вопросам моделирования синергетических эффектов от интеграционных процессов в экономике уделяется достаточное внимание в исследованиях отечественных авторов. Однако представленные модели преимущественно дают оценку лишь положительным эффектам от интеграции (диссинергия не учитывается). Для этого обычно используют функциональные выражения затрат на интеграцию и дополнительных выгод от нее с последующим стоимостным исчислением разницы между ними. Такие модели, бесспорно, являются полезными на предварительном этапе интеграции предпринимательских структур, однако никаким образом не отражают комплексные динамические (синергетические) риск-процессы в интеграционных структурах.

Проблемам же моделирования комплексного синергетического воздействия в риско- 
вой среде в отечественных научных работах уделено недостаточно внимания. По мнению автора, именно такая оценка имеет одно из ключевых значений для будущего развития интеграционных образований.

В контексте научного развития данной проблематики можно выделить исследования авторов 3.И. Абдулаевой и А. О. Недосекина, уделившим в своей работе достаточно большое внимание нечетко-множественному моделированию в инновационной рисковой среде [1], а также Р. А. Шамшилова, который на основе синергетического подхода и теории нечетких множеств разработал методику оценки инновационных рисков, учитывающую нелинейность в процессах инновационного развития [10].

Использование теории нечетких множеств также может находить широкое применение для развития вспомогательного аппарата механизма оценочно-прогнозного моделирования в интеграционных системах управления рисками. Так, в случае, когда математическая риск-модель не может быть представлена четкими параметрами, можно использовать треугольные нечеткие числа c соответствующей функцией принадлежности $\left(x_{\min }, x, x_{\max }-\right.$ нечеткий параметр $(X)$ приблизительно равен $x$ в диапазоне $\left[x_{\min }\right.$, $\left.\left.x_{\max }\right]\right)$. Тогда, преобразовав стандартное дифференциальное уравнение $(d x / d t)$ с учетом возможного синергетического риск-воздействия во времени получим общую формулу:

$$
\begin{gathered}
\frac{\partial x_{i}}{\partial t}+\frac{\partial x_{j}}{\partial t}+\ldots+\frac{\partial x_{n}}{\partial t}= \\
=f\left(x(\sin )_{\min }, x(\sin ), x(\sin )_{\max }\right),
\end{gathered}
$$

где $f\left(x(\sin )_{\min }, x(\sin ), x(\sin )_{\max }\right)$ - нечеткие динамические изменения совокупного (синергетического) воздействия $x_{i, j \ldots n}$ - риск-факторов во времени.

При этом при смене в формуле (1) временной функциональной компоненты (dt) на пространственную $(d z)$ получим функцию синергетических риск-процессов в пространственной распределительной системе. При замене функциональной компоненты $(d t$ либо $d z)$ также треугольным нечетким значением можно получить синергетическую функцию с определенными нечеткими временными и пространственными интервала- ми. Таким образом, полученное общее уравнение (модель) синергетических рисковых процессов формально представляет собой совокупность дифференциальных изменений взаимосвязанных риск-факторов во времени и пространстве, результатом которых является их функциональное синергетическое воздействие, выраженное нечетким треугольным числом.

Инструмент нечетких множеств относительно универсален и поэтому может комбинироваться с различными моделями синергетических риск-систем. Такое функциональное преобразование может способствовать прогнозированию сложных хаотических (синергетических) процессов в идентифицированных комплексных риск-системах. Однако, главным недостатком данного математического аппарата является значительная условность полученных прогнозных оценок, ведь при данной методике предельные минимальные и максимальные значения диапазона нечеткого числа также устанавливаются преимущественно при помощи экспертных оценок. Следовательно, экспертные ошибки при определении данного диапазона могут привести к некорректным результатам подобного моделирования, а слишком большое расширение диапазона предельных значений будет давать настолько условные результаты, что их практическое использование в управлении рисками станет невозможным.

Для более точных оценок комплексных синергетических рисковых взаимодействий (в том числе более точных количественных оценок предельных значений диапазонов параметров риск-системы), по мнению автора, необходимо развитие дополнительного математического аппарата, включающего инструментарий спектрального анализа колебаний и волн. В данном контексте исследования рисковая среда интегрированных предпринимательских структур будет представлять собой совокупность риск-сигналов на разных уровнях воздействия и взаимодействия.

Развитию такого подхода к оценке рисков могут способствовать исследования автора Б. А. Матвеева, который предлагает методику спектральной оценки рисков во времени (посредством анализа динамики поведения случайной функции $s(t))$, в основе которой лежит оценка формы энергетического спект- 
ра сигнала риска [7]. Комбинирование стандартного статистическо-математического инструментария (дисперсии, корреляции) с методологией спектрального анализа позволило данному исследователю выработать оригинальную методику прогнозной оценки реализации рисковых процессов.

По мнению автора, качественным развитием данного направления оценочнопрогнозного моделирования рисков может служить ее применение в контексте оценки рисковых колебаний во времени. При условии идентифицированных риск-систем с определенными математическими ожиданиями реализации риск-сигналов (в данном контексте - воздействий риск-факторов разных уровней $\left(s(t)_{i, y 1,2,3}, \ldots s(t)_{n, y 1,2,3}\right)$ во времени можно проводить сопоставимый анализ частоты, силы, амплитуды и синхронности этих колебаний. При этом комплексное сопоставление данных характеристик может позволить определить предельные значения их совокупного синергетического воздействия $\left(s(t)_{\text {sin, min }} ; s(t)_{\text {sin, max }}\right)$ в определенном временном интервале $T\left[t_{0}, \ldots t_{n}\right]$.

Преобразовав функцию

$$
T\left(s_{i, y 1,2,3}\right)=T\left(s_{i, y 1,2,3}\right)\left(A\left(s_{i, y 1,2,3}\right)\right),
$$

получим выражение:

$$
\begin{gathered}
T\left(s_{i, y 1,2,3}\right)\left(A\left(s_{i, y 1,2,3}\right)\right) \rightarrow \\
\rightarrow T\left(s_{j, y 1,2,3}\right)\left(A\left(s_{j, y 1,2,3}\right)\right) \rightarrow \ldots \rightarrow \\
\rightarrow T\left(s_{n, y 1,2,3}\right)\left(A\left(s_{n, y 1,2,3}\right)\right) \rightarrow \\
\rightarrow T\left(s_{\text {sin }}\right)\left(A\left(s_{\text {sin }}\right)\right),
\end{gathered}
$$

где $A$ - амплитуда, $T$ - временной период; $T\left(s_{\sin }\right)\left(A\left(s_{\sin }\right)\right)-$ функция периода амплитуды совокупного синергетического воздействия взаимосвязанных риск-сигналов $\left(s_{i, j, \ldots, n}\right)$.

Данное выражение отображает математическую модель нарастания синергетического воздействия риск-сигналов во времени в рамках определенного «релевантного периода» действия комплексной многоуровневой риск-системы.

При этом необходимо отметить, что, поскольку мы имеем дело с хаотическими динамически развивающимися системами, такое сопоставление нарастания амплитуд рискового воздействия дают лишь общие тенденции развития в «недолгосрочном релевантном» периоде. В случае же необ- ходимости долгосрочных перспективных формализованных оценок данный анализ синергетических риск-процессов должен быть дополнен инструментарием волновой теории.

Как известно, волны обладают импульсами и переносят энергию в процессе своего распространения с определенной скоростью. Даже при очень слабой нелинейности их амплитуда во времени будет нарастать, а спектр исходного возмущения будет расширяться в сторону больших частот [3]. Такое физическое поведение волн, по мнению автора, в полной мере отображает модель распространения нелинейных риск-сигналов в «долгосрочном релевантном периоде» в рамках развития многоуровневых риск-систем.

Тогда общий вид формулы групповой (совокупной) скорости волновых риск-процессов $\left(v_{\text {сов }}\right)$ будет иметь вид:

$$
v_{\mathrm{cOB}}=\frac{d \omega_{s}}{d k_{s}},
$$

где $d \omega_{s}$ - приращение частоты волновых колебаний риск-сигналов в группе (риск-системе); $d k_{s}$ - приращение волнового числа колебаний риск-сигналов в группе.

В этом случае общая функция темпов распространения совокупного синергетического риск-воздействия в «долгосрочном релевантном периоде» $\left(s_{\mathrm{sin}}\right)$ будет иметь вид:

$$
f\left(s_{\text {sin }}{ }^{\prime}\right)=T\left(s_{\text {sin }}\right)^{\prime}\left(A\left(s_{\text {sin }}\right)\right) \times v_{\text {сов }} \rightarrow \text { ТБ }(\Pi),
$$

где $T\left(s_{\sin }\right)^{\prime}\left(A\left(s_{\sin }\right)\right)$ - дифференцированная функция периода амплитуды волнового синергетического воздействия взаимосвязанных риск-сигналов; ТБ - точка бифуркации (полифуркации).

Таким образом, функция (4) показывает долгосрочные тенденции дифференцированного распространения синергетического риск-воздействия до достижения точки бифуркации (полифуркации) (полной трансформации риск-системы).

При этом дифференцированную функцию зависимости амплитуды колебаний синергетических риск-процессов $\left(A\left(s_{\mathrm{sin}}\right)\right)$ от совокупной скорости волновых риск-процессов можно представить так:

$$
A\left(s_{\mathrm{sin}}\right)=A\left(s_{\mathrm{sin}}\right)^{\prime}\left(v_{\text {сов }}\right) .
$$

Тогда общая функция силы распространения совокупного синергетического риск- 
воздействия в «долгосрочном релевантном периоде» $\left(s_{\sin }{ }^{\prime \prime}\right)$ будет иметь вид:

$$
\begin{gathered}
f\left(s_{\text {sin }}{ }^{\prime \prime}\right)=A\left(s_{\text {sin }}\right)^{\prime}\left(v_{\text {coв }}\right) \times \sum_{i=1}^{n \rightarrow \mathrm{TБ}} T_{i \ldots n}, \\
\sum_{i=1}^{n \rightarrow \mathrm{TБ}} T_{i \ldots n}-
\end{gathered}
$$

где

совокупность периодов полной волновой амплитуды колебаний синергетических рисквоздействий до достижения риск-системы точки бифуркации (полифуркации).

Следовательно, функция (6) соответственно отображает тенденции дифференцированного усиления (снижения) распространения синергетического риск-воздействия в «долгосрочном релевантном периоде».

По мнению автора, предложенные выше функциональные зависимости хоть и имеют общую форму, но могут служить эффективной базисной основой для стратегического оценочно-прогнозного моделирования синергетических процессов в комплексных рисксистемах, имеющего особую актуальность для расширенных интеграционных образований в контексте реализации парадигмы управления будущим посредством долгосрочного комплексного (стратегического) превентивного управления рисковой средой.

Заключение. Таким образом, представленные в работе направления развития вспомогательного аппарата содержат комплекс взаимодополняющих рекомендаций по повышению эффективности современных методов оценочно-прогнозного моделирования риск-систем, предложений по расширению вспомогательного инструментария в сторону получения актуальных для интеграционных систем риск-менеджмента оценочных прогнозов комплексных синергетических рискпроцессов.

Практический эффект от реализации данных направлений исследования в рамках внедрения интеграционных систем управления рисками интегрированных предпринимательских структур, сформированных на базе расширенных (кластерных) форм внутренней интеграции, имеет достаточно обширный спектр целевого назначения:

- представленный вспомогательный аппарат более эффективно выявляет рисковые взаимосвязи на разных уровнях воздействия с определением наиболее значимых рискогенерирующих факторов в системе, моделирует их динамические изменения и синергетические риск-процессы;

- представленные подходы моделирования комплексных риск-систем способствуют синтезу управления интеграционных систем управления рисками со всеми внутренними и внешними процессами, происходящими как непосредственно в самих интеграционных образованиях, так и в общей социальноэкономической сфере страны и регионов. Такой синтез позволяет осуществлять оценку и прогноз взаимодействия (взаимовлияния) макроэкономической среды и социальной сферы с микроэкономической средой (результатами деятельности интегрированных предпринимательских структур). Данный подход дает возможность, с одной стороны, минимизировать потери от возможных кризисных явлений макроуровня, а с другой позволяет выработать превентивные рычаги противодействия возникновению таких явлений посредством долгосрочного стратегического риск-ориентированного планирования и прогнозирования. Иными словами, реализация комплекса представленных направлений развития аппарата оценочно-прогнозного моделирования многоуровневых риск-систем может способствовать выработке сбалансированной модели управления интегрированными структурами в кратко-, средне- и долгосрочном периоде с оптимизацией всех значимых (релевантных) показателей, скорректированных на возможное динамическое (синергетическое) развитие рисковой среды;

- при глобальном развитии представленных подходов к моделированию рисковой среды посредством реализации новой парадигмы управления будущим на основе «тотального» внедрения комплексных многоуровневых интеграционных систем риск-менеджмента можно получить новую концептуальную модель коллективной национальной социально-экономической безопасности, позволяющей противостоять любым современным вызовам, в том числе глобального характера, которая в свою очередь даст возможность долгосрочного конкурентоспособного развития нацио- 
нальной экономики и социальной сферы в условиях растущей нестабильности и неопределенности мировой политэкономической среды.

\section{Литература}

1. Абдулаева 3. И., Недосекин А.О. Стратегический анализ инновационных рисков. - СПб: Изд-во Политехн. университета, 2013. - $150 \mathrm{c}$.

2. Алексеев А. П. Анализ синергетического эффекта интеграционного процесса производственных систем и методы его оценки // Современная экономика: проблемы и решения. - 2015. — №8 (68). - С. 8-14.

3. Безручко Б.П., Короновский А.А., Трубеиков Д. И., Храмов А.Е. Путь в синергетику. Экскурс в десяти лекциях. - М.: Книжный дом «Либроком», 2010. - 304 с.

4. Бондаренко А.И. Синергический эффект в управлении конкурентоспособностью предприятия // Евразийский Союз Ученых (ЕСУ). - 2014. - №4. - С. 15-17.

5. Иванов А.Е. Оценка синергетического эффекта в экономическом обосновании интеграции в промышленности. Автореферат. - Челябинск: Ю-УГУ, 2007. — 26 с.

6. Кузнецов Б.Л. Экономическая синергетика как методология экономического развития [Электронный ресурс]. C.34-36. - Режим доступа: http://www. docme.ru/doc/346991/34-b.-1.kuznecov-ekonomicheskaya-sinergetika-kak.

7. Матвеев Б.А. Теоретические основы спектрального метода измерения статистических рисков // Экономический анализ: теория и практика. - 2007. - №20 (101). C. 34-41.

8. Солошенко Р. В. Систематизация синергетических эффектов в экономике // Вестник Курской государственной сельскохозяйственной академии. - 2014. - №1. - С. 15-18.

9. Хасанова Г. Ф. Оценка эффекта синергии с помощью экономико-математических методов // Интернет-журнал Науковедение. - 2015. - №3 (28). - С. 1-10.

10. Шамшилов Р. А. Методика оценки эффективности инновационной деятельности предприятия с учетом рисков на основе синергетического подхода // Транспортное дело России. - 2010. - №1. - С. 15-18.

\section{References}

1. Abdulaeva Z.I., Nedosekin A.O. Strategicheskij analiz innovacionnyh riskov [Strategic analysis of innovative risks]. - SaintPetersburg: Izd-vo Politehn. universiteta, 2013. - 150 p.

2. Alekseev A.P. Analiz sinergeticheskogo jeffekta integracionnogo processa proizvodstvennyh sistem i metody ego ocenki [Analysis of the synergetic effect of the integration process of production systems and methods for its evaluation] // Sovremennaja jekonomika: problemy i reshenija [Modern economy: problems and solutions]. - 2015. - №8 (68). - Pp. 8-14.

3. Bezruchko B.P., Koronovskij A.A., Trubeckov D.I., Hramov A.E. Put' v sinergetiku. Jekskurs $\mathrm{v}$ desjati lekcijah [Path to synergetics. Excursus in ten lectures]. - Moscow: Knizhnyj dom «Librokom», 2010. - $304 \mathrm{p}$.

4. Bondarenko A.I. Sinergicheskij jeffekt v upravlenii konkurentosposobnost'ju predprijatija [Synergistic effect in enterprise competitiveness management] // Evrazijskij Sojuz Uchenyh (ESU) [Eurasian Union of Scientists (ESU)]. 2014. — №4. - Pp. 15-17.

5. Ivanov A.E. Ocenka sinergeticheskogo jeffekta $v$ jekonomicheskom obosnovanii integracii $\mathrm{v}$ promyshlennosti. Avtoreferat [Assessment of the synergetic effect in the economic justification of integration in industry. Autoabstract]. — Cheljabinsk: Ju-UGU, 2007. — 26 p.

6. Kuznecov B.L. Jekonomicheskaja sinergetika kak metodologija jekonomicheskogo razvitija [Economic synergetics as a methodology of economic development] [Jelektronnyj resurs]. - Pp. 34-36. - URL: http://www. docme.ru/doc/346991/34-b.-1.-kuznecov-e-konomicheskaya-sinergetika-kak.

7. Matveev B.A. Teoreticheskie osnovy spektral'nogo metoda izmerenija statisticheskih riskov [Theoretical foundations of the spectral method for measuring statistical risks] // Jekonomicheskij analiz: teorija i praktika [Economic analysis: theory and practice]. — 2007. №20 (101). - Pp. 34-41.

8. Soloshenko R. V. Sistematizacija sinergeticheskih jeffektovv jekonomike [Systematization of synergetic effects in the economy] // Vestnik Kurskoj gosudarstvennoj sel'skohozjajstvennoj akademii [Bulletin of the Kursk State agricultural Academy]. — 2014. — №1. — Pp. 15-18. 
9. Hasanova G.F. Ocenka jeffekta sinergii $\mathrm{s}$ pomoshh'ju jekonomiko-matematicheskih metodov [Evaluation of the synergy effect using economic and mathematical methods] // Internet-zhurnal Naukovedenie [The Internet journal of the sociology of Science]. - 2015. №3 (28). - Pp. 1-10.

10. Shamshilov R.A. Metodika ocenki jeffektivnosti innovacionnoj dejatel'nosti pred- prijatija s uchetom riskov na osnove sinergeticheskogo podhoda // Transportnoe delo Rossii [Methodology for evaluating the effectiveness of innovative activity of an enterprise taking into account risks based on a synergetic approach. Transport business of Russia]. — 2010. №1. - Pp. 15-18.

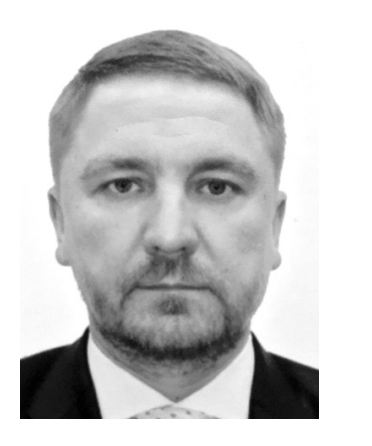

Корнеев Дмитрий Владимирович - кандидат экономических наук, директор Первого термометрового завода, г. Валдай, Россия.

Korneev Dmitry Vladimirovich - Candidate of Economic Sciences, Director, The First Thermometer Plant, Valdai, Russia.

117193, г. Москва, Новоясеневский пр., 25/20, кв. 173

25/20 Novoyasenevsky av., app. 173, 117193, Moscow, Russia

E-mail: director@1thermometer.ru 